\title{
Betti Posets and the Stanley Depth
}

\author{
Lukas Katthän 1
}

Received: 9 October 2015 / Revised: 19 December 2015 / Accepted: 4 February 2016 /

Published online: 15 February 2016

(C) Institute for Mathematical Sciences (IMS), Stony Brook University, NY 2016

\begin{abstract}
Let $S$ be a polynomial ring and let $I \subseteq S$ be a monomial ideal. In this short note, we propose the conjecture that the Betti poset of $I$ determines the Stanley projective dimension of $S / I$ or $I$. Our main result is that this conjecture implies the Stanley conjecture for $I$, and it also implies that sdepth $S / I \geq \operatorname{depth} S / I-$ 1. Recently, Duval et al. (A non-partitionable Cohen-Macaulay simplicial complex, arXiv:1504.04279, 2015) found a counterexample to the Stanley conjecture, and their counterexample satisfies sdepth $S / I=\operatorname{depth} S / I-1$. So if our conjecture is true, then the conclusion is best possible.
\end{abstract}

Keywords Monomial ideal · LCM-lattice · Betti poset · Stanley depth · Stanley conjecture

Mathematics Subject Classification Primary 05E40; Secondary 13D02 - 16W50

\section{Introduction}

Let $S$ be a polynomial ring and $I \subseteq S$ a monomial ideal. In this note we consider the Stanley depth of $S / I$ and of $I$, which is a combinatorial invariant. We refer the reader to Pournaki et al. (2009) for a short introduction to the subject and to Herzog (2013) for a comprehensive survey.

The lcm-lattice $L_{I}$ of a monomial ideal $I \subseteq S$ is the lattice of all least common multiples of subsets of the minimal generators of $I$. It is known that the isomorphism type of $L_{I}$ determines the projective dimension of $I$, cf. Gasharov et al. (1999). Further, the Betti poset $\mathcal{B}(I) \subset \mathbb{Z}^{n}$ is the poset of all multidegrees in which $S / I$ has non-

\footnotetext{
$凶 \quad$ Lukas Katthän

katthaen@math.uni-frankfurt.de

1 Institut für Mathematik, Goethe-Universität Frankfurt, 60054 Frankfurt am Main, Germany
} 
vanishing Betti numbers. It is known that the Betti poset is a subposet of $L_{I}$ and it is determined by the latter. Recently, Tchernev and Varisco (2015), and also Clark and Mapes (2014) showed that Betti poset already determines the projective dimension of $I$, in fact, it even determines the full structure of the minimal free resolution. In Ichim et al. (2014), Ichim, the author and Moyano Fernández showed that the Stanley projective dimension of $S / I$ and $I$ are determined by the isomorphism type of $L_{I}$ as well. Here, the Stanley projective dimension of a module $M$ can be defined as $\operatorname{spdim} M=\operatorname{dim} S-\operatorname{sdepth} M$. In the present paper, we propose the following extension of that result:

Conjecture 2.4 The Betti poset of a monomial ideal I determines the Stanley projective dimension of $S / I$ and $I$.

More precisely, if $I \subseteq S$ and $I^{\prime} \subseteq S^{\prime}$ are two monomial ideals in two polynomial rings $S$ and $S^{\prime}$ such that $\mathcal{B}(I) \cong \mathcal{B}\left(I^{\prime}\right)$, then it holds that $\operatorname{spdim}_{S} S / I=\operatorname{spdim}_{S^{\prime}} S^{\prime} / I^{\prime}$ and $\operatorname{spdim}_{S} I=\operatorname{spdim}_{S^{\prime}} I^{\prime}$.

The significance of this conjecture stems from the following result:

Theorem 3.1 If 2.4 is true, then for any monomial ideal $I \subset S$, it holds that

$$
\begin{aligned}
\text { sdepth } S / I & \geq \operatorname{depth} S / I-1 \text { and } \\
\text { sdepth } I & \geq \operatorname{depth} I .
\end{aligned}
$$

The original motivation for the research on the Stanley depth is the Stanley conjecture (Stanley 1982, Conjecture 5.2), which asserts that sdepth $M \geq$ depth $M$ for every $\mathbb{Z}^{n}$-graded finitely generated $S$-module $M$. Very recently, the Stanley conjecture was disproven by Duval et al. (2015). Indeed, these authors construct a monomial ideal I in some polynomial ring $S$, such that

$$
\text { sdepth } S / I=\text { depth } S / I-1 \text {. }
$$

Thus, our 2.4 would imply the Stanley conjecture for ideals, and it would give the best-possible bound for the Stanley depth of cyclic modules $S / I$.

We also show that 2.4 can be reduced to the following special case:

Conjecture 3.5 Let $I \subset S=\mathbb{K}\left[x_{1}, \ldots, x_{n}\right]$ be a squarefree monomial ideal, let further $I^{\prime}:=\left(I: x_{n}\right)$ and assume that $\mathcal{B}(I) \cong \mathcal{B}\left(I^{\prime}\right)$. Then it holds that sdepth $S / I=\operatorname{sdepth} S / I^{\prime}$, or equivalently $\operatorname{spdim} S / I=\operatorname{spdim} S / I^{\prime}$. Similarly, it holds that $\operatorname{sdepth} I=\operatorname{sdepth} I^{\prime}$, or equivalently $\operatorname{spdim} I=\operatorname{spdim} I^{\prime}$.

This note is structured as follows. In Sect. 2 we review some background necessary for stating 2.4. Also, we add some remarks about this conjecture. In the subsequent Sect. 3 we provide the proof of Theorem 3.1 and of the equivalence of 2.4 with 3.5. Finally, in Sect. 3.3 we show that a weak version of 2.4 holds for generic ideals.

\section{Statement of the Conjecture}

Throughout the paper, let $\mathbb{K}$ denote some fixed field. By $S$ and $S^{\prime}$ we denote polynomial rings over $\mathbb{K}$, which we always consider with the fine grading. 


\subsection{The Stanley Depth}

Consider the polynomial ring $S=\mathbb{K}\left[x_{1}, \ldots, x_{n}\right]$ endowed with the fine $\mathbb{Z}^{n}$-grading. Let $M$ be a finitely generated (multi-)graded $S$-module, and let $m \in M$ be a homogeneous element. Let $Z \subset\left\{x_{1}, \ldots, x_{n}\right\}$ be a subset of the set of indeterminates of $S$. The $\mathbb{K}[Z]$-submodule $m \mathbb{K}[Z]$ of $M$ is called a Stanley space of $M$ if $m \mathbb{K}[Z]$ is a free $\mathbb{K}[Z]$-module. A Stanley decomposition of $M$ is a finite family

$$
\mathcal{D}=\left(\mathbb{K}\left[Z_{i}\right], m_{i}\right)_{i \in \mathcal{I}}
$$

in which $Z_{i} \subset\left\{x_{1}, \ldots, x_{n}\right\}$ and $m_{i} \mathbb{K}\left[Z_{i}\right]$ is a Stanley space of $M$ for each $i \in \mathcal{I}$ with

$$
M \cong \bigoplus_{i \in \mathcal{I}} m_{i} \mathbb{K}\left[Z_{i}\right]
$$

as a multigraded $\mathbb{K}$-vector space. This direct sum carries the structure of an $S$-module and has therefore a well-defined depth. The Stanley depth of $M$, sdepth $M$, is defined to be the maximal depth of a Stanley decomposition of $M$. Similarly, the Stanley projective dimension spdim $M$ of $M$ is defined as the minimal projective dimension of a Stanley decomposition of $M$. Note that

$$
\operatorname{spdim} M+\operatorname{sdepth} M=n
$$

by the Auslander-Buchsbaum formula.

In the sequel, we will concentrate on modules which are either cyclic $S / I$ or ideals $I \subset S$. In this case, Herzog et al. (2009) provide a convenient alternative description of the Stanley depth in terms of interval partitions. Note that there is no known relation between $\operatorname{spdim} S / I$ and $\operatorname{spdim} I$.

\subsection{The Lcm-Lattice and the Betti Poset}

Let $I \subset S$ be a monomial ideal. The lcm-lattice $L_{I}$ of $I$ is the lattice of all least common multiples of subsets of the minimal generators of $I$, together with a minimal element $\hat{0}$.

The following two results by Gasharov, Peeva and Welker, resp. Ichim, the author and Moyano Fernández connect the lcm-lattice with projective dimension and the Stanley projective dimension.

Theorem 2.1 Let $I \subset S$ and $I^{\prime} \subset S^{\prime}$ be two monomial ideals. If there exists a surjective join-preserving map $L_{I} \rightarrow L_{I^{\prime}}$, then (Gasharov et al. 1999; Ichim et al. 2014)

$\operatorname{pdim} S^{\prime} / I^{\prime} \leq \operatorname{pdim} S / I$, and

$\operatorname{spdim} S^{\prime} / I^{\prime} \leq \operatorname{spdim} S / I$. 
The corresponding statements hold as well for $I$ and $I^{\prime}$ instead of $S / I$ and $S^{\prime} / I^{\prime}$. In particular, the isomorphism type of $L_{I}$ determines both the projective dimension and the Stanley projective dimension of both $S / I$ and I.

Here, $\operatorname{pdim} M$ denotes the projective dimension of $M$. For any given finite atomistic lattice $L$, one can find a monomial ideal $I \subseteq S$ in some polynomial ring such that $L \cong$ $L_{I}$, cf. Phan (2005); Mapes (2013); Ichim et al. (2014). The preceding theorem thus implies that the invariants $\operatorname{pdim}_{\mathrm{Q}} L:=\operatorname{pdim} S / I, \operatorname{pdim}_{\mathrm{I}} L:=\operatorname{pdim} I, \operatorname{spdim}_{Q} L:=$ spdim $S / I$ and $\operatorname{spdim}_{\mathrm{I}} L:=\operatorname{spdim} I$ do not depend on the choice of $I$. The subscripts $\mathrm{Q}$ and I stand for "quotient" and "ideal", respectively.

We denote by $\beta_{i, m}^{S}(S / I):=\operatorname{dim}_{\mathbb{K}} \operatorname{Tor}_{i}^{S}(S / I, \mathbb{K})_{m}$ the multigraded Betti number of $S / I$ over $S$ in homological degree $i$ and multidegree $m$. It is known that the Betti numbers can be computed in terms of the lcm-lattice by the following formula, cf. (Gasharov et al. 1999, Theorem 2.1):

$$
\beta_{i, m}^{S}(S / I)= \begin{cases}\operatorname{dim}_{\mathbb{K}} \tilde{H}_{i-2}\left(L_{<m} ; \mathbb{K}\right) & \text { if } m \in L_{I}, \\ 0 & \text { otherwise }\end{cases}
$$

Here, $\tilde{H}_{i-2}\left(L_{<m} ; \mathbb{K}\right)$ denotes the reduced simplicial homology of the order complex of $L<m \backslash\left\{\hat{0}_{L}\right\}=\left\{n \in L \backslash\left\{\hat{0}_{L}\right\}: n<m\right\}$. Motivated by this formula, the Betti poset was introduced in Clark and Mapes (2014).

Definition 2.2 Let $L$ be a finite atomistic lattice. The Betti poset of $L$ is the subset

$$
\mathcal{B}(L):=\left\{m \in L: \tilde{H}_{i-2}\left(L_{<m} ; \mathbb{K}\right) \neq 0 \text { for some } i\right\}
$$

Note that $\mathcal{B}(L)$ might depend on $\mathbb{K}$. If $I \subseteq S$ is a monomial ideal, then we set $\mathcal{B}(I):=\mathcal{B}\left(L_{I}\right)$.

It turns out that the Betti poset of a monomial ideal contains the same homological information about the ideal as the lcm-lattice:

Theorem 2.3 [Theorem 5.3 of Tchernev and Varisco (2015), Theorem 2.1 of Clark and Mapes (2014)] The Betti poset $\mathcal{B}(I)$ of a monomial ideal $I \subseteq S$ determines the structure of the minimal free resolution of $S / I$. In particular, it determines the Betti numbers and the projective dimension of $S / I$.

Given these results, it seems natural to ask whether the part of 2.1 concerning the Stanley projective dimension also extends to the Betti poset:

Conjecture 2.4 The Betti poset of a monomial ideal I determines the Stanley projective dimension of $S / I$ and $I$.

More precisely, if $I \subseteq S$ and $I^{\prime} \subseteq S^{\prime}$ are two monomial ideals in two polynomial rings $S$ and $S^{\prime}$ such that $\mathcal{B}(I) \cong \mathcal{B}\left(I^{\prime}\right)$, then it holds that $\operatorname{spdim}_{S} S / I=\operatorname{spdim}_{S^{\prime}} S^{\prime} / I^{\prime}$ and $\operatorname{spdim}_{S} I=\operatorname{spdim}_{S^{\prime}} I^{\prime}$. 
Remark 2.5 (1) 2.4 seems a natural conjecture to us, and we have some evidence for it. Nevertheless, we are far from being convinced that this conjecture really holds. Moreover, it is possible that 2.4 holds for $S / I$ but not for $I$, or vice versa. In the sequel, all statements about quotients $S / I$ depend only on the part of 2.4 concerning quotients, and similarly all statements about ideals $I$ depend only on the other part of 2.4 .

(2) We know from Ichim et al. (2016) that pdim $S / I=\operatorname{spdim} S / I$ for all ideals with up to five generators. Hence 2.4 holds for quotients of those ideals. Similarly, using the complete enumeration of lcm-lattices of ideals with four generators in Ichim et al. (2016), we verified 2.4 for ideals with up to four generators.

(3) As mentioned above, the counterexample to the Stanley conjecture by Duval et al satisfies sdepth $S / I=$ depth $S / I-1$. Given Theorem 3.1 below, one could try to amplify the defect to also obtain a counterexample to 2.4. One possibility would be to consider $S / I \otimes_{\mathbb{K}} S / I$. However, while the depth is additive under this operation, the Stanley depth is only superadditive, i.e.,

$$
\text { sdepth } M \otimes_{\mathbb{K}} N \geq \operatorname{sdepth} M+\text { sdepth } N
$$

for $S$-modules $M, N$, see (Bruns et al. 2010, Proposition 2.10) or also (Rauf 2010, Theorem 3.1). So this does not immediately yields counterexamples to our conjecture.

\section{Discussion of the Conjecture}

\subsection{An Important Consequence}

In this section we prove the following result.

Theorem 3.1 If 2.4 is true, then for any monomial ideal $I \subset S$, it holds that

$$
\begin{aligned}
\text { sdepth } S / I & \geq \operatorname{depth} S / I-1 \text { and } \\
\text { sdepth } I & \geq \operatorname{depth} I .
\end{aligned}
$$

Before we give the proof of Theorem 3.1, we collect some statements that we will use. If $L$ is atomistic lattice and $a \in L$, then the rank of $a$ is the number of atoms below it. Further, recall that an element $a \in L$ is called meet-irreducible if it cannot be written as a meet of two elements $b, c$ which are distinct from $a$. If $a \in L$ is meetirreducible, then the subposet $L \backslash\{a\}$ is again a lattice. The following is a special case of Katthän (2015, Lemma 6.4).

Lemma 3.2 Let $p \in \mathbb{N}$, L be a finite atomistic lattice and $a \in L$ meet-irreducible. If rk $a<2 p$, then it holds that $\operatorname{spdim}_{\mathrm{I}} L \leq \max \left\{p, \operatorname{spdim}_{\mathrm{I}} L \backslash\{a\}\right\}$.

Recall that the length $\ell=\ell(L)$ of a finite poset $L$ equals the maximal length of a strictly ascending chain $l_{0}<l_{1}<\cdots<l_{\ell}$ in $L$. 
Theorem 3.3 [Corollary 2.5, Katthän and Seyed Fakhari (2015)] For a finite atomistic lattice L, it holds that

$$
\begin{aligned}
& \operatorname{spdim}_{\mathrm{Q}} L \leq \ell(L) \text { and } \\
& \operatorname{spdim}_{\mathrm{I}} L \leq \ell(L)-1 .
\end{aligned}
$$

The following proposition summarizes several results of Katthän (2015) in a form which is suitable for the present purpose.

Proposition 3.4 Let $I \subseteq S$ be a monomial ideal and let $p:=\operatorname{pdim} S / I$. Then there exists a monomial ideal $I^{\prime} \subseteq S^{\prime}$ in some polynomial ring $S^{\prime}$ which satisfies the following properties:

(1) $\operatorname{pdim} S^{\prime} / I^{\prime}=\operatorname{pdim} S / I$ and (thus) $\operatorname{pdim} I^{\prime}=\operatorname{pdim} I$,

(2) $\operatorname{spdim} S^{\prime} / I^{\prime} \geq \operatorname{spdim} S / I$ and $\operatorname{spdim} I^{\prime} \geq \operatorname{spdim} I$,

(3) the length of $\mathcal{B}\left(I^{\prime}\right)$ is $p$, and finally

(4) $\mathcal{B}\left(I^{\prime}\right)$ is the face poset of an acyclic simplicial complex.

Proof Let $k$ be the number of generators of $I$. Consider the set $\mathcal{L}$ of isomorphism classes of atomistic lattices with $k$ atoms. This is a finite poset, where the order is given by setting $L \geq L^{\prime}$ if there is a surjective join-preserving map $L \rightarrow L^{\prime}$. Consider the subposet $\mathcal{L}(p) \subseteq \mathcal{L}$ of all lattices $L$ such that $\operatorname{pdim}_{\mathrm{Q}} L=p$. Clearly, $L_{I} \in \mathcal{L}(p)$. As this is a finite poset, we can find a maximal element $L^{\prime} \in \mathcal{L}(p)$ with $L^{\prime} \geq L_{I}$. Such a lattice is called maximal in Katthän (2015). Let further $I^{\prime} \subset S^{\prime}$ be a monomial ideal with $L_{I^{\prime}}=L^{\prime}$. By construction, it holds that $\operatorname{pdim} S^{\prime} / I^{\prime}=\operatorname{pdim} S / I$, and by Theorem 2.1 it holds that $\operatorname{spdim} S^{\prime} / I^{\prime} \geq \operatorname{spdim} S / I$ and $\operatorname{spdim} I^{\prime} \geq \operatorname{spdim} I$.

For the remaining parts of the claim we recall the description of the maximal lattices from Katthän (2015). By the Theorems 4.3 and 4.5 of Katthän (2015), there exists a $(p-1)$-dimensional simplicial complex $\Delta$ on $k$ vertices whose $(p-2)$-skeleton is complete and which is $\mathbb{K}$-acyclic, such that

$$
L^{\prime} \cong\left\{F \subseteq[k]:\left.\Delta\right|_{F} \text { is } \mathbb{K} \text {-acyclic }\right\}
$$

Further, the Betti poset of $I^{\prime}$ coincides with the face poset of $\Delta$ (cf. Katthän 2015 , Corollary 4.4). So the last claim follows and for the penultimate claim we note that $\ell\left(\mathcal{B}\left(I^{\prime}\right)\right)=\operatorname{dim} \Delta+1=p$.

Proof of Theorem 3.1 Let $p:=\operatorname{pdim} S / I$. We may replace the ideal $I$ by the ideal $I^{\prime}$ of Proposition 3.4 without changing the validity of the claim. Let $L^{\prime}:=\mathcal{B}\left(I^{\prime}\right) \cup\{\hat{1}\}$, where $\hat{1}$ is a new maximal element. By part (4) of Proposition $3.4, L^{\prime}$ is an atomistic lattice with $\mathcal{B}\left(L^{\prime}\right)=\mathcal{B}\left(I^{\prime}\right)$. So we can find another monomial ideal $I^{\prime \prime} \subset S^{\prime \prime}$ in some polynomial ring with $L_{I^{\prime \prime}}=L^{\prime}$. By our assumption on 2.4 , it follows that $\operatorname{spdim} S^{\prime} / I^{\prime}=\operatorname{spdim} S^{\prime \prime} / I^{\prime \prime}$ and $\operatorname{spdim} I^{\prime}=\operatorname{spdim} I^{\prime \prime}$.

Note that the length of $L^{\prime}$ equals $p+1$. Hence, using Theorem 3.3 we can conclude that

$$
\operatorname{spdim} S^{\prime} / I^{\prime}=\operatorname{spdim} S^{\prime \prime} / I^{\prime \prime} \leq \ell\left(L^{\prime}\right)=p+1=\operatorname{pdim} S^{\prime} / I^{\prime}+1
$$


So the claim for $S^{\prime} / I^{\prime}$ is proven.

It remains to show the claim for $I^{\prime}$. If $p=2$, then it holds that sdepth $I^{\prime} \geq \operatorname{depth} I^{\prime}$ by Katthän (2015), Corollary 7.2 (see also Katthän and Seyed Fakhari 2015, Lemma 4.3). So we may assume that $p>2$. We will use 3.2. For this, note that $L^{\prime}$ is a graded poset of rank $p+1$. Hence every element $a \in L^{\prime}$ of rank $p$ is meet-irreducible. Further, $p>2$ implies that $\operatorname{rk} a=p<2(p-1)$.

So we conclude with 3.2 that $\operatorname{spdim}_{\mathrm{I}} L^{\prime} \leq \max \left(p-1, \operatorname{spdim}_{\mathrm{I}} L^{\prime} \backslash\{a\}\right)$. Iterating this procedure, we can remove all elements of rank $p$ from $L^{\prime}$ and obtain a lattice $\tilde{L}$ of length $p$. In conclusion, we have that

$$
\begin{aligned}
\operatorname{spdim} I^{\prime} & =\operatorname{spdim}_{\mathrm{I}} L^{\prime} \leq \max \left(p-1, \operatorname{spdim}_{\mathrm{I}} \tilde{L}\right) \\
& \leq \max (p-1, \ell(\tilde{L})-1)=p-1=\operatorname{pdim} I^{\prime} .
\end{aligned}
$$

\subsection{An Explicit Version of the Conjecture}

We believe that the following more explicit formulation might be helpful in proving 2.4 .

Conjecture 3.5 Let $I \subset S=\mathbb{K}\left[x_{1}, \ldots, x_{n}\right]$ be a squarefree monomial ideal, let further $I^{\prime}:=\left(I: x_{n}\right)$ and assume that $\mathcal{B}(I) \cong \mathcal{B}\left(I^{\prime}\right)$. Then it holds that sdepth $S / I=\operatorname{sdepth} S / I^{\prime}$, or equivalently $\operatorname{spdim} S / I=\operatorname{spdim} S / I^{\prime}$. Similarly, it holds that $\operatorname{sdepth} I=\operatorname{sdepth} I^{\prime}$, or equivalently $\operatorname{spdim} I=\operatorname{spdim} I^{\prime}$.

Proposition 3.6 3.5 is equivalent to 2.4.

The construction of $M(B)$ in the following proof is taken from Section 6 of Tchernev and Varisco (2015).

Proof 3.5 is clearly a special case of 2.4 , so we only need to prove one implication.

Let $I \subseteq S$ be a monomial ideal and set $L:=L_{I}$. Denote by $A \subset L$ the set of atoms of $\bar{L}$ and let further $\Sigma(A)$ be the boolean algebra on $A$. There is an injective meet-preserving map $j: L \rightarrow \Sigma(A)$, which maps an element to the set of atoms below it. We consider $L$ as a subset of $\Sigma(A)$ via $j$.

Let $B:=j(\mathcal{B}(I))$ and let further $M(B) \subset \Sigma(A)$ be the set of all meets in $\Sigma(A)$ of subsets of $B$. Here, we consider the maximal element of $\Sigma(A)$ as the meet of the empty set. Then $M(B)$ is an atomistic lattice (Tchernev and Varisco 2015, Lemma 6.1), and the inclusion $M(B) \subseteq L$ preserves the meet. Further, it holds that $\mathcal{B}(M(B))=B$ by Tchernev and Varisco (2015, Proposition 6.5).

We order the elements $a_{1}, \ldots, a_{r}$ of $L \backslash M(B)$ be decreasing rank and set $L_{i}:=$ $L \backslash\left\{a_{1}, \ldots, a_{i}\right\}$. This way, we obtain an increasing chain

$$
M(B)=L_{r} \subsetneq L_{r-1} \subsetneq \cdots \subsetneq L_{0}=L
$$

of lattices, where all the inclusions are meet-preserving (cf. Katthän 2015, Lemma 3.7). It is easy to see by induction on $i$ that $\mathcal{B}\left(L_{i}\right)=\mathcal{B}(L)$ for all $i$. Indeed, this is obvious 
for $i=0$. For $i>0$, it holds that $a_{i+1} \notin \mathcal{B}(L)=\mathcal{B}\left(L_{i}\right)$, and hence the arguments given in Lemma 3.8 and Remark 3.9 of Katthän (2015) show that $\mathcal{B}\left(L_{i+1}\right)=\mathcal{B}\left(L_{i}\right)$.

Fix an $i$ such that $0 \leq i \leq r$. It follows from Ichim et al. (2014, Theorem 3.4) that there exists a squarefree monomial ideal $J \subset S^{\prime \prime}$ in some polynomial ring $S^{\prime \prime}$ and a variable $x \in S^{\prime \prime}$, such that $L_{i} \cong L_{J}$ and $L_{i+1} \cong L_{(J: x)}$. Thus, 3.5 implies that $\operatorname{spdim}_{\mathrm{Q}} L_{i}=\operatorname{spdim}_{\mathrm{Q}} L_{i+1}$ and $\operatorname{spdim}_{\mathrm{I}} L_{i}=\operatorname{spdim}_{\mathrm{I}} L_{i+1}$. As this holds for all $i$, we arrive at the conclusion that

$$
\operatorname{spdim} S / I=\operatorname{spdim}_{\mathrm{Q}} L=\operatorname{spdim}_{\mathrm{Q}} M(B)
$$

and

$$
\operatorname{spdim} I=\operatorname{spdim}_{\mathrm{I}} L=\operatorname{spdim}_{\mathrm{I}} M(B) .
$$

Now let $I^{\prime} \subset S^{\prime}$ be a second monomial ideal with $\mathcal{B}(I) \cong \mathcal{B}\left(I^{\prime}\right)$. This clearly implies that $M(\mathcal{B}(I)) \cong M\left(\mathcal{B}\left(I^{\prime}\right)\right)$, and hence that

$$
\operatorname{spdim} S / I=\operatorname{spdim}_{\mathrm{Q}} M(\mathcal{B}(I))=\operatorname{spdim}_{\mathrm{Q}} M\left(\mathcal{B}\left(I^{\prime}\right)\right)=\operatorname{spdim} S^{\prime} / I^{\prime}
$$

and similar for spdim $I$.

Remark 3.7 (1) Note that the inequalities $\operatorname{sdepth}_{S} S / I \leq \operatorname{sdepth}_{S} S / I^{\prime}$ and also sdepth $I \leq$ sdepth $I^{\prime}$ are clear, because every Stanley decomposition of $S / I$ and $I$ restricts to a Stanley decomposition of $S / I^{\prime}$ and $I^{\prime}$, respectively. So the difficulty is to extend a Stanley decomposition of $S / I^{\prime}$ or $I^{\prime}$ to a Stanley decomposition of $S / I$ or $I$.

(2) For 3.1, it would be enough to prove 3.5 (and thus 2.4) for those ideals which actually appear in the proof of Theorem 3.1. In particular, one may assume that the minimal free resolution of $I$ is supported on the Scarf complex, and that the latter is a stoss complex in the sense of Katthän (2015), i.e. an acyclic $(p-1)$-dimensional simplicial complex with a complete $(p-2)$-skeleton, where $p=\operatorname{pdim} S / I$. Note that such a resolution is a truncation of the Taylor resolution.

(3) The assumption that $I$ is squarefree is inessential, as it does not affect the $1 \mathrm{~cm}$ lattices. One may further assume in 3.5 that all generators of $I$ have the same degree, cf. (Ichim et al. 2014, Proposition 5.12).

It seems desirable to understand the implications of the condition $\mathcal{B}(I) \cong \mathcal{B}\left(I^{\prime}\right)$ in 3.5. One possible approach is to consider the Hilbert series of $S / I$. Recall that it is given by

$$
\mathbf{H}\left(S / I ; t_{1}, \ldots, t_{n}\right)=\frac{1}{\left(1-t_{1}\right) \cdots\left(1-t_{m}\right)} \sum_{m \in \mathbb{Z}^{n}} t^{m} \sum_{i \geq 0}(-1)^{i} \beta_{i, m}(S / I),
$$

where we write $t^{m}=t_{1}^{m_{1}} \cdots t_{n}^{m_{n}}$. Now the condition $\mathcal{B}(I) \cong \mathcal{B}\left(I^{\prime}\right)$ implies that the Hilbert series of $S / I^{\prime}$ has the same "shape", in the sense that no further cancellation of terms occurs. As Stanley decompositions can be seen a decompositions of the Hilbert 
series, this might imply that the Stanley decompositions are also similar. However, the following example shows that it is not enough to consider this "shape" of the Hilbert series.

Example 3.8 Let $S=\mathbb{K}[a, b, c, x, y]$ and consider the following two ideals

$$
\begin{aligned}
& I_{1}:=\left(a^{2} x^{2}, b^{2} x^{2}, c^{2} x^{2}, a^{2} b^{2} c^{2}, a b c x y\right) \\
& I_{2}:=\left(a^{2} x^{2}, b^{2} x^{2}, c^{2} x^{2}, a^{2} b^{2} c^{2}, a b c x\right)
\end{aligned}
$$

in $S$. One can easily compute that $\operatorname{pdim} S / I_{1}=4$ and that $\operatorname{pdim} S / I_{2}=3$. Further, both ideals have only five generators, so their Stanley projective dimensions coincide with the respective projective dimensions (Ichim et al. 2016). In particular, their Betti posets are nonisomorphic and their Stanley projective dimensions differ. Further, using the algorithm of Ichim and Zarojanu (2014), we computed that $\operatorname{spdim} I_{1}=\operatorname{spdim} I_{2}$.

On the other hand, their Hilbert series have the same "shape". The reason is that for all elements $m \in \mathcal{B}\left(I_{1}\right) \backslash \mathcal{B}\left(I_{2}\right)$ it holds that $\sum_{i \geq 0}(-1)^{i} \beta_{i, m}\left(S / I_{1}\right)=0$.

\subsection{Generic Ideals}

In this section we show that a weak version of 2.4 holds for generic ideals in the sense of Miller et al. (2000).

Proposition 3.9 Let $I \subset S$ be a generic monomial ideal. Let further $I^{\prime} \subseteq S^{\prime}$ be a further monomial ideal, such that there is a surjective join-preserving map $L_{I} \rightarrow L_{I^{\prime}}$ and assume that $\mathcal{B}(I) \cong \mathcal{B}\left(I^{\prime}\right)$.

Then it holds that $\operatorname{spdim}_{S} S / I=\operatorname{spdim}_{S^{\prime}} S^{\prime} / I^{\prime}$.

The addition assumption that there is a map $L_{I} \rightarrow L_{I^{\prime}}$ is not a severe restriction, because in our proof of Theorem 3.1 we only consider this situation.

Proof Let $p=\operatorname{pdim}_{S} S / I$. Recall that the Scarf complex of $I$ is the subset $\Delta(I) \subseteq L_{I}$ of those elements which can be written as a join of atoms in a unique way. In general, the Betti poset contains the Scarf complex, and in the generic situation these two coincide. So every element of the Betti poset of $I$ is a join of atoms in a unique way. But then this also holds for $I^{\prime}$, and hence $\mathcal{B}\left(I^{\prime}\right)=\Delta\left(I^{\prime}\right)$ as well. In particular, there exists an element $a \in \Delta\left(I^{\prime}\right)$ of rank $p=\operatorname{pdim}_{S} S / I=\operatorname{pdim}_{S^{\prime}} S^{\prime} / I^{\prime}$. We can construct a surjective join-preserving map from $L_{I^{\prime}}$ to a boolean algebra on $p$ atoms by sending every element $b \in L_{I^{\prime}}$ to the set of atoms below $b \wedge a$. The boolean algebra on $p$ atoms can be considered as lcm-lattice of an ideal $J$ generated by $p$ variables, and the latter has Stanley projective dimension $p$. Hence we conclude with Theorem 2.1 that $\operatorname{spdim}_{S^{\prime}} S^{\prime} / I^{\prime} \geq p$. On the other hand, the Stanley conjecture holds for $S / I$ (as $I$ is

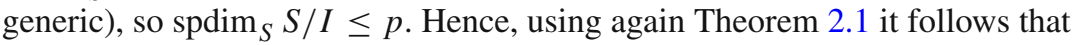

$$
p \leq \operatorname{spdim}_{S^{\prime}} S^{\prime} / I^{\prime} \leq \operatorname{spdim}_{S} S / I \leq p,
$$

so the claim follows. 


\section{References}

Bruns, W., Krattenthaler, C., Uliczka, J.: Stanley decompositions and Hilbert depth in the Koszul complex. J. Commut. Algebra 2(3), 327-357 (2010)

Clark, T.B., Mapes, S.: The Betti poset in monomial resolutions. In: Preprint (2014). arXiv:1407.5702

Clark, T.B., Mapes, S.: Rigid monomial ideals. J. Commut. Algebra 6(1), 33-51 (2014)

Duval, A.M., Goeckner, B., Klivans, C.J., Martin, J.L.: A non-partitionable Cohen-Macaulay simplicial complex. In: Preprint (2015). arXiv:1504.04279

Gasharov, V., Peeva, I., Welker, V.: The LCM-lattice in monomial resolutions. Math. Res. Lett. 6, 521-532 (1999)

Herzog, J.: A survey on Stanley depth. In: Monomial Ideals, Computations and Applications, pp. 3-45. Springer (2013)

Herzog, J., Vladoiu, M., Zheng, X.: How to compute the Stanley depth of a monomial ideal. J. Algebra 322(9), 3151-3169 (2009)

Ichim, B., Katthän, L., Moyano Fernández, J.J.: Lcm-lattices and Stanley depth: a first computational approach. Exp. Math. 25(1):46-53 (2016). arXiv:1408.4255

Ichim, B., Katthän, L., Moyano Fernández, J. J.: Stanley depth and the lcm-lattice. In: Preprint (2014). arXiv: 1405.3602

Ichim, B., Zarojanu, A.: An algorithm for computing the multigraded Hilbert depth of a module. Exp. Math 23(3), 322-331 (2014)

Katthän, L., Seyed Fakhari, S.A.: Two lower bounds for the Stanley depth of monomial ideals. Mathematische Nachrichten. 288(11-12):1360-1370 (2015). arXiv:1405.5450

Katthän, L.: Stanley depth and simplicial spanning trees. J. Algebraic Comb. 42(2): 507-536 (2015). arXiv: 1410.3666

Mapes, S.: Finite atomic lattices and resolutions of monomial ideals. J. Algebra 379, 259-276 (2013)

Miller, E., Sturmfels, B., Yanagawa, K.: Generic and cogeneric monomial ideals. J. Symb. Comput. 29(4), 691-708 (2000)

Phan, J.: Minimal monomial ideals and linear resolutions. In: Preprint (2005). arXiv:math/0511032

Pournaki, M., Seyed Fakhari, S., Tousi, M., Yassemi, S.: Stanley depth? In: Notices of the AMS 56.9 (2009)

Rauf, A.: Depth and Stanley depth of multigraded modules. Commun. Algebra 38(2), 773-784 (2010)

Stanley, R.: Linear Diophantine equations and local cohomology. Invent. Math 68, 175-193 (1982)

Tchernev, A., Varisco, M.: Modules over categories and Betti posets of monomial ideals. In: Proceedings of the AMS143 (2015), pp. 5113-5128. arXiv:1308.1434 